\title{
(6) OPEN ACCESS \\ The role of specific visual subfields in collisions with oncoming cars during simulated driving in patients with advanced glaucoma
}

\author{
Shiho Kunimatsu-Sanuki, ${ }^{1,2}$ Aiko Iwase, ${ }^{3}$ Makoto Araie, ${ }^{4}$ Yuki Aoki, ${ }^{2}$ Takeshi Hara, ${ }^{2,5}$ \\ Takeo Fukuchi, ${ }^{6}$ Sachiko Udagawa, ${ }^{7}$ Shinji Ohkubo, ${ }^{7}$ Kazuhisa Sugiyama, ${ }^{7}$ \\ Chota Matsumoto, ${ }^{8}$ Toru Nakazawa, ${ }^{1}$ Takuhiro Yamaguchi, ${ }^{9}$ Hiroshi Ono ${ }^{10}$
}

For numbered affiliations see end of article.

\section{Correspondence to} Dr Shiho Kunimatsu-Sanuki, Department of Ophthalmology, Tohoku University Graduate School of Medicine, 1-1 Seiryocho Aoba-ku Sendai-shi Miyagiken 980-8574, Japan; shihok-tky@umin.ac.jp

Received 22 March 2016 Revised 28 September 2016 Accepted 29 September 2016 Published Online First 17 October 2016

\section{CrossMark}

To cite: Kunimatsu-Sanuki $\mathrm{S}$, Iwase A, Araie M, et al. $\mathrm{Br} J$ Ophthalmol 2017;101:896-901.

\section{ABSTRACT}

Background/aims To assess the role of specific visual subfields in collisions with oncoming cars during simulated driving in patients with advanced glaucoma. Methods Normal subjects and patients with glaucoma with mean deviation $<-12 \mathrm{~dB}$ in both eyes (Humphrey Field Analyzer 24-2 SITA-S program) used a driving simulator (DS; Honda Motor, Tokyo). Two scenarios in which oncoming cars turned right crossing the driver's path were chosen. We compared the binocular integrated visual field (IVF) in the patients who were involved in collisions and those who were not. We performed a multivariate logistic regression analysis; the dependent parameter was collision involvement, and the independent parameters were age, visual acuity and mean sensitivity of the IVF subfields.

Results The study included 43 normal subjects and 100 patients with advanced glaucoma. And, 5 of the 100 patients with advanced glaucoma experienced simulator sickness during the main test and were thus excluded. In total, 95 patients with advanced glaucoma and 43 normal subjects completed the main test of DS. Advanced glaucoma patients had significantly more collisions than normal patients in one or both DS scenarios $(p<0.001)$. The patients with advanced glaucoma who were involved in collisions were older $(p=0.050)$ and had worse visual acuity in the better eye $(p<0.001)$ and had lower mean IVF sensitivity in the inferior hemifield, both $0^{\circ}-12^{\circ}$ and $13^{\circ}-24^{\circ}$ in comparison with who were not involved in collisions $(p=0.012$ and $p=0.034)$. A logistic regression analysis revealed that collision involvement was significantly associated with decreased inferior IVF mean sensitivity from $13^{\circ}$ to $24^{\circ}(p=0.041)$, in addition to older age and lower visual acuity $(p=0.018$ and $p<0.001)$. Conclusions Our data suggest that the inferior hemifield was associated with the incidence of motor vehicle collisions with oncoming cars in patients with advanced glaucoma.

\section{INTRODUCTION}

An adequate visual field is one of the most basic requirements for drivers for safe driving in addition to visual acuity. Patients with visual field defects have difficulty safely assessing traffic, following other vehicles, staying in their lane, noticing stoplights, hazards, pedestrians or other vehicles on the road and performing other driving tasks. ${ }^{1}{ }^{2}$ Progressive optic neuropathy in diseases such as glaucoma often cause various types of visual field defect that do not affect central visual acuity. ${ }^{3}$ Many patients with visual field defects, even those with severe defects, are thus able to pass visual acuity tests and receive or retain their driving licences. However, these patients have an increased risk of motor vehicle collisions (MVCs), as has been shown in previous studies based on real-world collisions. $^{4-11}$

Although each MVC has different circumstances, such as the failure to notice traffic signals or stop signs, the sudden appearance of children or vehicles, or vehicles turning into the path of the driver, the degree of this risk may depend on the specific area of the visual field that is affected. Furthermore, every patient with glaucoma suffers from a unique pattern of visual field defects. ${ }^{12}$ It is therefore challenging to obtain clear information on the individual risks faced by drivers with glaucoma. In fact, previous studies, including our own preliminary study, have yielded conflicting results on which areas of the visual field are related to MVC involvement. ${ }^{11} 13-15$

The key merit of studies based on driving simulator (DS) systems, in comparison with studies based on real-world MVC history and on-road driving, is that they include collisions. This allows us to directly investigate the factors associated with the collisions. DS systems have been used in previous studies to investigate the influence of visual field defects on driving ability, ${ }^{16-19}$ and the reliability of these systems has been confirmed by comparing their results with those obtained from on-road testing. ${ }^{20}{ }^{21}$ Moreover, DS systems can investigate the effects of different configurations of visual field defects on the ability to avoid MVCs under standardised conditions. In this study, which included a large number of patients, we performed testing with a single, particularly dangerous type of driving scenario, in which an oncoming car at an intersection turned into the driver's path. Thus, taking advantage of the controlled conditions of a DS, this study investigated the role of specific visual subfields in a single type of collision.

\section{MATERIALS AND METHODS \\ Subjects}

We included normal subjects and patients with advanced glaucoma, diagnosed as mean deviation (MD) values in both eyes of $<-12 \mathrm{~dB}$, measured with the Humphrey Visual Field Analyzer Swedish Interactive Threshold Algorithm standard 24-2 
(HFA 24-2, Carl Zeiss Meditec, Dublin, California, USA). All participants were active drivers who had driven within at least the previous three months. All patients were examined at the Department of Ophthalmology of Jichi Medical University, the Tajimi Iwase Eye Clinic, the Department of Ophthalmology of Kanazawa University and the Department of Ophthalmology of Niigata University between September 2010 and July 2012 under identical conditions. To minimise bias arising from age, only subjects younger than 70 years old were included.

Before using the DS, all participants completed a questionnaire to determine their (1) age and sex, (2) driving habits and history (years since acquisition of first driving licence, time spent driving per week and motor vehicle accident (MVA) involvement over the previous 5 years) and (3) current illnesses and medical history. They also had a complete ophthalmological examination, including best-corrected visual acuity, a slit-lamp examination, intraocular pressure measurement with Goldmann applanation tonometry, gonioscopy, a stereoscopic fundus examination and standard automated perimetry with the HFA 24-2. We excluded those with cataract or other non-glaucoma ocular diseases that could cause visual field defects after a basic ophthalmic examination. We also excluded patients with a history of systemic or neurological disease that could reduce the ability to drive or could affect the visual field, based on self-reporting during patient interviews. Eyes with unreliable visual field results (fixation loss $>20 \%$, false-positive rate $>15 \%$ or falsenegative rate $>33 \%$ ) were also excluded.

\section{Integrated visual field}

The binocular integrated visual field (IVF) was calculated by merging the results of monocular HFA 24-2 tests from each eye using the points with the higher sensitivity from each test. ${ }^{22} 23$ Fifty-two test points were included, each representing a $6^{\circ} \times 6^{\circ}$ IVF subfield.

In the advanced glaucoma group, we evaluated mean IVF sensitivity in the central area of the inferior and superior hemifields within $0^{\circ}-12^{\circ}\left(\mathrm{IVF}_{0-12}\right)$ and within $13^{\circ}-24^{\circ}\left(\mathrm{IVF}_{13-24}\right)$ of the fixation point. ${ }^{24}$ We also divided the IVF within $0^{\circ}-12^{\circ}$ of the fixation point into six clusters $\left(\mathrm{VF}_{\mathrm{A}}, \mathrm{B}, \mathrm{C}, \mathrm{D}, \mathrm{E}, \mathrm{F}\right)$ (figure 1). ${ }^{24} \mathrm{We}$

superior hemifield (from 13 to 24 degrees): superior IVF $_{13-24}$

superior hemifield (from 0 to 12 degrees): superior IVF

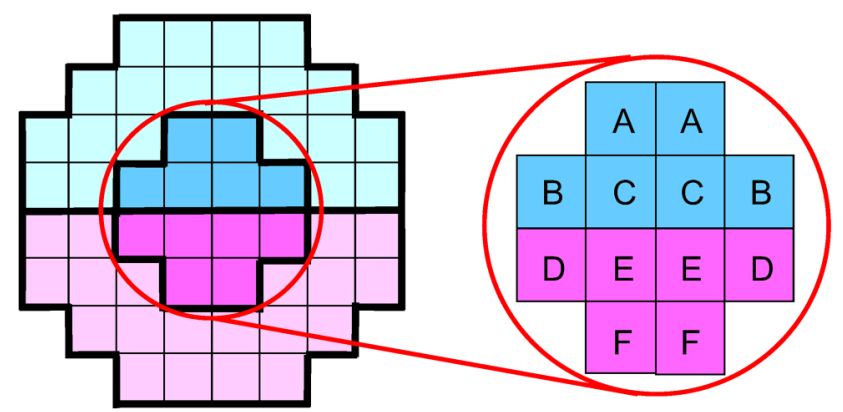

inferior hemifield (from 0 to 12 degrees): inferior $I V F_{0-12}$ inferior hemifield (from 13 to 24 degrees): inferior IVF $_{13-24}$

Figure 1 Test point clusters (A-F) located within the central $0^{\circ}-12^{\circ}$. The location of integrated visual field (IVF) test points was within $0^{\circ}-12^{\circ}$ $\left(\mathrm{IVF}_{0-12}\right)$ and $13^{\circ}-24^{\circ}\left(\mathrm{IVF}_{13-24}\right)$ of the fixation point in both the superior and inferior hemifields. The test points within $0^{\circ}-12^{\circ}$ were divided into six clusters $\left(\mathrm{VF}_{\mathrm{A}}, \mathrm{VF}_{\mathrm{B}}, \mathrm{VF}_{\mathrm{C}}, \mathrm{VF}_{\mathrm{D}}, \mathrm{VF}_{\mathrm{E}}\right.$ and $\left.\mathrm{VF}_{\mathrm{F}}\right)$. then compared mean sensitivity in each IVF area and each IVF cluster in the patients who were and were not involved in collisions.

\section{Driving simulator}

The DS, previously described in detail, ${ }^{13}$ used a revised version of the Honda Safety Navi (Honda Motor, Tokyo) system (righthand drive version). The examinees undertook a 2 min practice session followed by the $5 \mathrm{~min}$ main test. The only requirement was for the examinee to brake when he or she felt it was necessary. If an examinee reported feeling simulator sickness during the practice session, further testing was immediately abandoned. The main test contained 14 scenarios depicting such situations as coming to a stop sign, traffic light or road hazard, or a child suddenly rushing out in front of the car. In this study, we focused on two scenarios depicting oncoming right-turning cars at an intersection. We recorded the number of collisions in these two scenarios. Figure 2 shows a screenshot of the simulation. The yellow line shows the hazard's track.

\section{Statistical analysis}

Differences in demographic characteristics between the normal subjects and patients with glaucoma, as well as between the patients with glaucoma who were or were not involved in collisions, were determined using the t-test, $\chi^{2}$ test and the Mann-Whitney U test. We also performed a multivariate logistic regression analysis in the patients with advanced glaucoma. The dependent parameter was collision involvement, and the independent parameters were age, visual acuity and mean sensitivity of the IVF subfields (analysis 1: the superior and inferior $\mathrm{IVF}_{0-12}$; analysis 2: the superior and inferior $\left.I \mathrm{VF}_{13-24}\right)$. All statistical analyses were made with JMP V.9.0. $\mathrm{p}$ Values $<0.05$ were considered statistically significant.

\section{RESULTS}

The study included 43 normal subjects and 100 patients with advanced glaucoma. None of the normal subjects experienced simulator sickness. Also, 5 of the 100 patients with advanced glaucoma completed the practice session but experienced simulator sickness during the main test and were excluded. In total, 43 normal subjects (26 males and 17 females) and 95 patients with glaucoma (66 males and 29 females) completed the main test of the DS. There were no differences between the advanced glaucoma and normal control groups in sex or driving history, including hours of driving exposure and number of MVAs in the previous 5 years. The patients with advanced glaucoma were older than the normal subjects $(p=0.022)$ and had more years of driving experience $(p=0.048)$ (table 1$)$. The proportion of subjects who were involved in at least one collision was significantly higher in the advanced glaucoma group (76 of 95 patients; $80 \%$ ) than the normal group (11 of 43 subjects; 26\%) in the two DS scenarios with oncoming right-turning cars $\left(\chi^{2}\right.$ test, $\left.\mathrm{p}<0.001\right)$.

Among the normal subjects, there were no significant differences between the subjects involved in one or more collisions $(n=11)$ or no collisions $(n=32)$ in age, sex, driving history (including driving experience, hours of driving exposure and number of MVAs in the previous 5 years), visual acuity or MD value. On the other hand, the patients with advanced glaucoma who were involved in one or more collisions $(n=76)$ were older $(\mathrm{p}=0.050)$ and had worse visual acuity in the better eye than those who were not involved in collisions $(n=19)(\mathrm{p}<0.001)$. The mean sensitivity of the inferior $\mathrm{IVF}_{0-12}$ and inferior $\mathrm{IVF}_{13-24}$ was also significantly lower in the patients with glaucoma involved in collisions than those not involved in collisions 
Figure 2 Screenshot of a simulated scenario. In the screenshots, the yellow line indicates the track of the hazard across the image. The driver's vehicle had a simulated speed of $50 \mathrm{~km} / \mathrm{hour}$. A blue car ahead of the vehicle turns right into its path. The car appears $10^{\circ}$ right of centre and moves left.

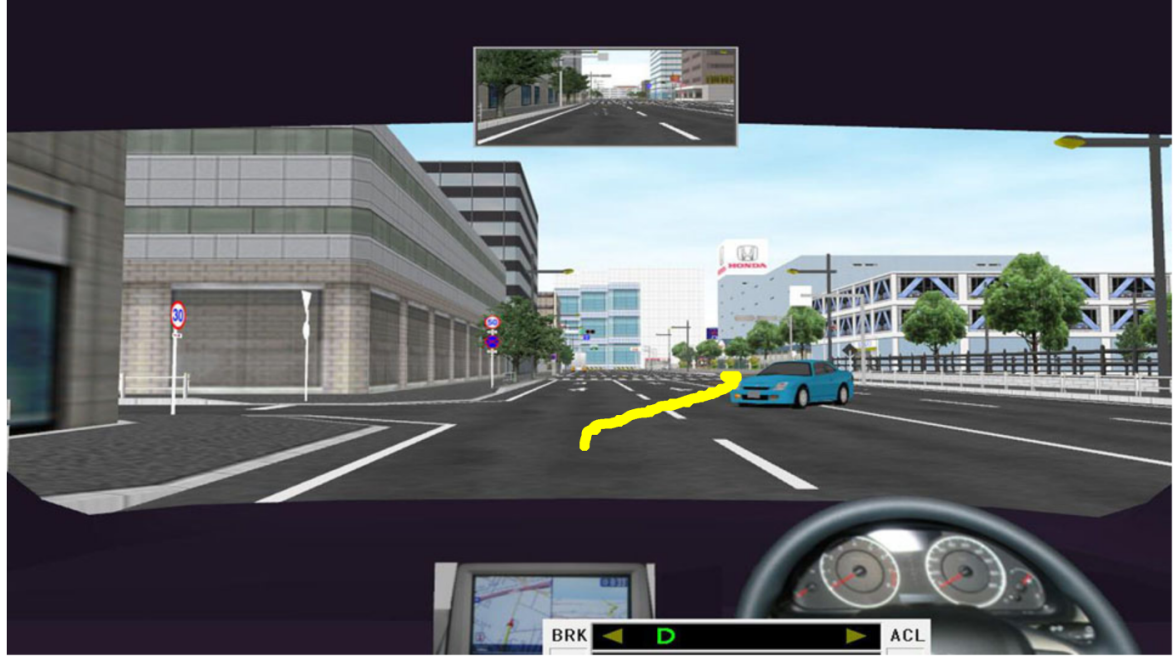

( $p=0.012$ and $p=0.034$, respectively) (table 2). Our analysis in the patients with advanced glaucoma of IVF clusters within $0^{\circ}-$ $12^{\circ}$ of the fixation point showed that there were clear differences between the collision-involved and collision-uninvolved patients in mean sensitivity in $\operatorname{VF}_{\mathrm{E}}(\mathrm{p}=0.024)$ and $\mathrm{VF}_{\mathrm{F}}$ $(p=0.041)$. On the other hand, there were no significant differences between the two groups in $\mathrm{VF}_{\mathrm{D}}$ and in the superior paracentral clusters, that is, $\mathrm{VF}_{\mathrm{A}}, \mathrm{VF}_{\mathrm{B}}$ and $\mathrm{VF}_{\mathrm{C}}$ (table 2).

We performed two separate logistic regression analyses in the patients with advanced glaucoma for different areas of the IVF. Analysis 1 showed that collision involvement was not significantly associated with mean sensitivity in the superior and inferior $\mathrm{IVF}_{0-12}(\mathrm{p}=0.860, \mathrm{p}=0.112)$, although it was associated with age $(p=0.017$; OR $1.099 ; 95 \%$ CI 1.016 to 1.202$)$ and better-eye VA

Table 1 Comparison of demographic, driving and vision characteristics of study participants by group

\begin{tabular}{|c|c|c|c|}
\hline Characteristics & $\begin{array}{l}\text { Normal control } \\
(n=43)\end{array}$ & $\begin{array}{l}\text { Glaucoma } \\
(n=95)\end{array}$ & p Value \\
\hline \multicolumn{4}{|l|}{ Demographic } \\
\hline Age (years) & $53.8 \pm 8.5$ & $57.4 \pm 7.7$ & $0.022^{\dagger}$ \\
\hline Gender (male/female) & $26 / 17$ & $66 / 29$ & $0.298^{\dagger}$ \\
\hline \multicolumn{4}{|l|}{ Driving } \\
\hline Driving years & $30.8 \pm 9.8$ & $34.3 \pm 8.5$ & $0.048^{*}$ \\
\hline Driving exposure (hours/week) & $9.9 \pm 11.0$ & $6.5 \pm 7.2$ & $0.074^{*}$ \\
\hline Number of MVAs by group & $5(11.3 \%)$ & $17(17.9 \%)$ & $0.455^{\ddagger}$ \\
\hline \multicolumn{4}{|l|}{ Visual acuity } \\
\hline Better-eye logMAR & $-0.04 \pm 0.08$ & $-0.08 \pm 0.03$ & $<0.001^{*}$ \\
\hline Worse-eye logMAR & $-0.07 \pm 0.07$ & $0.17 \pm 0.38$ & $<0.001^{*}$ \\
\hline \multicolumn{4}{|l|}{ HFA 24-2 MD (dB) } \\
\hline Better-eye MD & $0.36 \pm 1.0$ & $-18.19 \pm 4.7$ & $<0.001 \S$ \\
\hline Worse-eye MD & $-0.34 \pm 1.1$ & $-22.63 \pm 4.9$ & $<0.001 \S$ \\
\hline \multicolumn{4}{|l|}{ IVF sensitivity (dB) } \\
\hline Superior & $31.0 \pm 1.3$ & $13.9 \pm 7.3$ & $<0.001 \S$ \\
\hline Inferior & $32.0 \pm 1.0$ & $18.1 \pm 7.0$ & $<0.001 \S$ \\
\hline \multicolumn{4}{|c|}{$\begin{array}{l}\text { Values are mean } \pm S D \text {. } \\
{ }^{*} p \text { indicates unpaired t-test. } \\
t p \text { indicates } \chi^{2} \text { test. } \\
\neq p \text { indicates Fisher's exact test. } \\
\S p \text { indicates Mann-Whitney U test. } \\
\text { HFA, Humphrey Visual Field Analyzer; IVF, integrated visual field; logMAR, logarithm } \\
\text { of the minimum angle of resolution; MD, mean deviation; MVA, motor vehicle } \\
\text { accident. }\end{array}$} \\
\hline
\end{tabular}

(10 logarithm of the minimum angle of resolution (logMAR), $\mathrm{p}<0.001$; OR $28.59 ; 95 \%$ CI 3.44 to 754.85 ) (table 3). Analysis 2 showed that collision involvement was significantly associated with mean sensitivity in the inferior $\operatorname{IVF}_{13-24}(\mathrm{p}=0.041$; OR 0.910 ; $95 \%$ CI 0.815 to 0.996$)$, as well as age $(\mathrm{p}=0.018$; OR $1.10 ; 95 \%$ CI 1.02 to 1.20$)$ and better-eye VA $(10 \log$ MAR, $\mathrm{p}<0.001$; OR $75.71 ; 95 \%$ CI 6.62 to 2669.40 ) (table 3).

\section{DISCUSSION}

In this study, we attempted to investigate the association of visual field defects with collisions with oncoming right-turning cars by testing patients with advanced glaucoma in a DS. Our results indicated that lower mean sensitivity in the inferior IVF hemifield contributed significantly to MVCs with oncoming right-turning cars in a DS.

Recently, a number of studies have described the importance of determining which areas of the visual field are associated with MVC involvement. Huisingh et $a l^{14}$ reported that drivers with a severely impaired lower or left field were more involved in MVCs, while Glen et $a l^{15}$ found that lowered performance in the hazard perception test (a part of UK driving examinations) was more strongly associated with defects in the upper visual field than the lower field. On the other hand, Yuki et al ${ }^{12}$ reported that central VF damage had no effect on MVCs; these studies have yielded conflicting results on which areas of the VF are most likely to be associated with MVCs. In our study, normal subjects who were involved in collisions had no significant differences in age with the subjects who were not involved. However, the subjects with advanced glaucoma had differences in age, visual acuity and visual field sensitivity. Comparing IVF clusters in the patients with advanced glaucoma who were involved and uninvolved in collisions showed that mean sensitivity in the inferior clusters within $12^{\circ}$ of the fixation point, and the overall mean sensitivity of the inferior $\mathrm{IVF}_{13-24}$, may have contributed to the subjects' increased involvement in MVCs with oncoming right-turning cars. A logistic regression analysis in the advanced glaucoma revealed that in the DS scenario showing oncoming right-turning cars the number of collisions during simulated driving was significantly associated with decreased inferior $\mathrm{IVF}_{13-24}$ mean sensitivity in addition to older age and worse visual acuity.

We focused on scenarios in which an oncoming car at an intersection turned into the driver's path because this situation can be very serious. The importance of focusing on specific 
Table 2 Comparison of demographic, driving and vision characteristics of collision-involved and collision-uninvolved patients

\begin{tabular}{|c|c|c|c|c|c|c|}
\hline & \multicolumn{2}{|c|}{ Normal control $(n=43)$} & \multirow[b]{3}{*}{ p Value } & \multirow{2}{*}{\multicolumn{2}{|c|}{$\begin{array}{l}\text { Glaucoma }(n=95) \\
\text { Collisions in DS }\end{array}$}} & \multirow[b]{3}{*}{ p Value } \\
\hline & \multicolumn{2}{|c|}{ Collisions in DS } & & & & \\
\hline & No $(n=32)$ & Yes $(n=11)$ & & No $(n=19)$ & Yes $(n=76)$ & \\
\hline \multicolumn{7}{|l|}{ Demographic } \\
\hline Age (years) & $53.8 \pm 8.0$ & $53.2 \pm 9.6$ & $0.804^{*}$ & $53.9 \pm 8.4$ & $58.2 \pm 7.3$ & $0.050^{*}$ \\
\hline Sex (male/female) & $20 / 12$ & $5 / 6$ & $0.480 t$ & $13 / 6$ & $54 / 22$ & $0.786 t$ \\
\hline \multicolumn{7}{|l|}{ Driving } \\
\hline Driving experience (years) & $30.8 \pm 9.9$ & $30.7 \pm 10.1$ & $0.990^{*}$ & $32.8 \pm 9.1$ & $34.6 \pm 8.3$ & 0.441 * \\
\hline Driving exposure time (hours/week) & $9.8 \pm 10.2$ & $6.0 \pm 7.8$ & $0.526^{*}$ & $9.8 \pm 10.2$ & $6.0 \pm 7.8$ & $0.116^{*}$ \\
\hline Number of MVAs & $3(9.4 \%)$ & $2(18.1 \%)$ & $0.589 t$ & $4(21.1 \%)$ & $13(17.1 \%)$ & $0.740 \dagger$ \\
\hline \multicolumn{7}{|l|}{ Mean visual acuity (logMAR) } \\
\hline Better eye & $-0.08 \pm 0.02$ & $-0.07 \pm 0.03$ & $0.240 *$ & $-0.09 \pm 0.04$ & $-0.03 \pm 0.08$ & $<0.001^{*}$ \\
\hline Worse eye & $-0.07 \pm 0.03$ & $-0.03 \pm 0.12$ & $0.358^{*}$ & $0.17 \pm 0.42$ & $0.17 \pm 0.38$ & $0.978^{*}$ \\
\hline \multicolumn{7}{|l|}{ HFA 24-2 mean deviation (dB) } \\
\hline Better eye & $0.36 \pm 1.0$ & $-0.19 \pm 0.8$ & $0.133 \ddagger$ & $-16.2 \pm 3.3$ & $-18.70 \pm 4.9$ & $0.055 \ddagger$ \\
\hline Worse eye & $-0.34 \pm 1.1$ & $-1.0 \pm 1.0$ & $0.132 \ddagger$ & $-21.1 \pm 4.5$ & $-23.01 \pm 5.0$ & $0.132 \ddagger$ \\
\hline \multicolumn{7}{|l|}{ IVF mean sensitivity $(\mathrm{dB})$} \\
\hline Superior $\mathrm{IVF}_{0-12}$ & $33.1 \pm 0.7$ & $32.4 \pm 1.3$ & $0.318 \ddagger$ & $15.3 \pm 9.6$ & $12.2 \pm 9.3$ & $0.221 \neq$ \\
\hline Inferior $\mathrm{IVF}_{0-12}$ & $31.5 \pm 0.9$ & $32.7 \pm 1.4$ & $0.340 \ddagger$ & $23.9 \pm 6.1$ & $18.6 \pm 8.8$ & $0.012 \ddagger$ \\
\hline Superior $\mathrm{IVF}_{13-24}$ & $30.3 \pm 1.3$ & $29.6 \pm 0.9$ & $0.205 \ddagger$ & $15.5 \pm 8.0$ & $13.9 \pm 7.3$ & $0.500 \ddagger$ \\
\hline Inferior IVF $_{13-24}$ & $31.6 \pm 0.9$ & $31.3 \pm 1.1$ & $0.589 \ddagger$ & $20.7 \pm 6.2$ & $16.9 \pm 7.4$ & $0.034 \ddagger$ \\
\hline \multicolumn{7}{|l|}{ IVF sectors mean sensitivity $(\mathrm{dB})$} \\
\hline $\mathrm{VF}_{\mathrm{A}}$ & & & & $9.2 \pm 10.9$ & $9.0 \pm 10.8$ & $0.772 \ddagger$ \\
\hline $\mathrm{VF}_{\mathrm{B}}$ & & & & $15.5 \pm 12.1$ & $12.0 \pm 10.6$ & $0.237 \ddagger$ \\
\hline $\mathrm{VF}_{\mathrm{C}}$ & & & & $21.3 \pm 9.2$ & $15.7 \pm 11.5$ & $0.096 \ddagger$ \\
\hline $\mathrm{VF}_{\mathrm{D}}$ & & & & $24.2 \pm 6.9$ & $20.1 \pm 9.6$ & $0.086 \neq$ \\
\hline $\mathrm{VF}_{\mathrm{E}}$ & & & & $27.3 \pm 6.3$ & $22.1 \pm 9.8$ & $0.024 \ddagger$ \\
\hline $\mathrm{VF}_{\mathrm{F}}$ & & & & $20.2 \pm 9.9$ & $13.6 \pm 11.7$ & $0.041 \neq$ \\
\hline
\end{tabular}

Table 3 Multivariate logistic regression analysis of factors influencing the risk of collisions with oncoming cars during simulated driving, shown as OR with $95 \% \mathrm{Cl}$ (model 1)

\begin{tabular}{|c|c|c|c|}
\hline Independent variable & $\mathrm{OR}^{*}$ & $95 \% \mathrm{Cl}$ & p Value \\
\hline \multicolumn{4}{|l|}{ Analysis 1} \\
\hline Age (years) & 1.10 & $1.02-1.20$ & 0.017 \\
\hline Better-eye 10 logMAR & 28.59 & $3.44-754.85$ & $<0.001$ \\
\hline Superior $\mathrm{IVF}_{0-12}(\mathrm{~dB})$ & 1.01 & $0.94-1.07$ & 0.860 \\
\hline Inferior $\mathrm{IVF}_{0-12}(\mathrm{~dB})$ & 0.94 & $0.85-1.01$ & 0.112 \\
\hline \multicolumn{4}{|l|}{ Analysis 2} \\
\hline Age (years) & 1.10 & $1.02-1.20$ & 0.018 \\
\hline Better-eye 10 logMAR & 75.71 & $6.62-2669.40$ & $<0.001$ \\
\hline Superior $\operatorname{IVF}_{12-24}(\mathrm{~dB})$ & 1.04 & $0.95-1.15$ & 0.347 \\
\hline Inferior $\mathrm{IVF}_{12-24}(\mathrm{~dB})$ & 0.91 & $0.82-0.99$ & 0.041 \\
\hline
\end{tabular}

IVF, integrated visual field; logMAR, logarithm of the minimum angle of resolution.

scenarios is suggested by the existence of laws to prevent elderly people with dementia from driving. For example, the Japanese government recently established a law requiring drivers older than 75 years to undergo cognitive testing if they commit certain characteristic types of traffic violation, such as ignoring signals or driving the wrong way on expressways. There is thus a growing recognition that certain accident types may serve as indicators of specific types of driver impairment. Therefore, validating the contribution of visual field impairment in individuals who are involved in characteristic types of traffic violation (ie, collisions with oncoming cars) is thought to be useful in identifying at-risk subjects in the real-world MVCs.

A strength of this study was the inclusion of patients with advanced glaucoma (MD worse than -12). Many studies have suggested that patients with glaucoma, particularly those with advanced damage, have a greater risk of MVC, but to the best of our knowledge, only one ${ }^{25}$ has included patients with advanced glaucoma. Driving cessation is an effective way of avoiding the risks associated with visual field loss, but it also negatively affects quality of life and the ability to live with independence. It is therefore important to correctly assess the impact of visual impairment due to glaucoma on the ability of patients to drive safely. Our results suggest that in patients with advanced glaucoma the inferior hemifield IVF was most associated with MVCs with oncoming cars. In glaucoma, the lower central field often remains relatively preserved until the very late stages of the disease. ${ }^{26}$ The patients with glaucoma in our study followed this pattern: they had better IVF sensitivity in the inferior hemifield than in the superior hemifield. Furthermore, our results showed that in these patients the greatest potential risk of a particular type of MVC, those that occur with oncoming right-turning cars in a DS, was associated with decreases in inferior IVF sensitivity. Thus, our study confirms the importance 
of the inferior IVF hemifield in patients with advanced glaucoma who continue driving.

Our study had several limitations. First, using a DS has a number of inherent limitations. ${ }^{2}$ The scenarios shown in the DS differed from real-world situations in ways that may have inflated the number of collisions. Moreover, our study used only one specific type of DS scenario rather than a variety of scenarios showing different situations. Nevertheless, our results were consistent with those of Huisingh et al, ${ }^{14}$ who studied the relationship between MVC involvement and the areas of the visual field used while driving, and found that severe impairment of the lower or left field was associated with MVC involvement. Thus, we believe that our study is a valuable first step towards future, more precise investigations of patterns of visual field impairment that affect the ability to drive safely.

Second, the patients with advanced glaucoma involved in one or more collisions tended to be older than those not involved in collisions $(58.2 \pm 7.3$ vs $53.9 \pm 8.4$ years, $\mathrm{p}=0.0503)$. It is possible that factors associated with increasing age (eg, dementia, age-related decrease in vision, mild cataracts) might have confounded this result. However, our study excluded subjects $>70$ years of age in order to minimise effects of these factors. Furthermore, because the univariate analysis revealed a significant association between collision involvement and the inferior IVF, we performed a multivariable logistic regression analysis to separately analyse the influences of age, visual acuity and visual field damage on collisions with oncoming cars in simulated driving. Our results suggested that older patients with glaucoma with more advanced glaucomatous damage in the inferior hemifield and lower visual acuity might benefit from advice to be particularly cautious of oncoming cars. Identification of factors associated with advanced age ( $>70$ years) awaits further study.

Third, in contrast to previous studies that used eye-tracking devices to study driving performance, our study used an assumed fixation point. However, we used a scenario in which an oncoming car at an intersection turned into the driver's path, which is a type of MVC that occurs while the driver is looking ahead. Thus, we consider that eye movements only minimally influenced our results. Nevertheless, an eye-tracking device will be necessary in future studies including other scenarios, such as hazards coming from the side.

In conclusion, we assessed the relationship of specific visual subfield defects with collisions with an oncoming car, under the controlled conditions provided by a DS, in patients with advanced glaucoma. We found that the inferior visual field, as well as age and visual acuity, was an important factor associated with the ability to safely navigate these specific collision scenarios. Our findings may be useful in creating guidelines for safe driving for patients with glaucoma and for the development of clear warning systems to encourage risky drivers to reconsider driving and avoid potential MVAs.

\footnotetext{
Author affiliations

${ }^{1}$ Department of Ophthalmology, Tohoku University Graduate School of Medicine, Miyagi, Japan

${ }^{2}$ Department of Ophthalmology, Jichi Medical University, Tochigi, Japan

${ }^{3}$ Tajimi Iwase Eye Clinic, Gifu, Japan

${ }^{4}$ Kanto Central Hospital of the Mutual Aid Association of Public School Teachers, Tokyo, Japan

${ }^{5}$ Hara Eye Hospital, Tochigi, Japan

${ }^{6}$ Department of Ophthalmology, Niigata University, Niigata, Japan

${ }^{7}$ Department of Ophthalmology, Kanazawa University Graduate School of Medical Science, Ishikawa, Japan

${ }^{8}$ Department of Ophthalmology, Kinki University Faculty of Medicine, Osaka, Japan ${ }^{9}$ Division of Biostatistics, Tohoku University Graduate School of Medicine, Miyagi, Japan

${ }^{10}$ Honda Motor Co., Tokyo, Japan
}

Acknowledgements The authors thank Mr Tim Hilts for editing the language, and Masahiro Kami, MD, PhD (Division of Social Communication System for Advanced Clinical Research, Institute of Medical Science, University of Tokyo, Tokyo), Shigeaki Kato, PhD (Sendai Kousei Hospital, Miyagi), Makoto Itoh (Department of Risk Engineering, SIE, University of Tsukuba, Ibaraki) and Tomoyuki Sanuki, PhD (Department of Physics, Tohoku University, Miyagi) for providing assistance with the design and analysis of the study. None of these persons received any compensation for their contributions. This article was presented as a poster at the American Academy of Ophthalmology Annual Meeting, November 2013, New Orleans, Louisiana (chosen as best poster).

Contributors All the authors included in this paper fulfill the criteria of authorship. SK-S designed the study and wrote the manuscript, had full access to all of the data in the study and takes responsibility for the integrity of the data and the accuracy of the data analysis. Al, YA, TH, SO, SU and TF designed the study and collected the data. MA designed the study, interpreted the data and reviewed and edited the manuscript. CM and TN made critical revisions to the manuscript for intellectual content. TY took part in analysing and interpreting the data. KS contributed to proofing of the paper. HO did administrative, technical or material support.

Funding This work was supported by Japan Society for the Promotion of Science KAKENHI grant number 26462630 .

Competing interests $\mathrm{HO}$ is an employee of Honda Motor.

\section{Patient consent Obtained.}

Ethics approval The Ethics Committee of Jichi Medical University approved the research at all institutions (rinA10-62). All aspects of the protocol conformed to the tenets of the Declaration of Helsinki.

Provenance and peer review Not commissioned; externally peer reviewed.

Open Access This is an Open Access article distributed in accordance with the Creative Commons Attribution Non Commercial (CC BY-NC 4.0) license, which permits others to distribute, remix, adapt, build upon this work non-commercially, and license their derivative works on different terms, provided the original work is properly cited and the use is non-commercial. See: http://creativecommons.org/ licenses/by-nc/4.0/

\section{REFERENCES}

1 Owsley C, McGwin G. Vision and driving. Vision Res 2010;50:2348-61.

2 Owsley C, Wood JM, McGwin G. A roadmap for interpreting the literature on vision and driving. Surv Ophthalmol 2015;60:250-62.

3 Weinreb RN, Aung T, Medeiros FA. The pathophysiology and treatment of glaucoma a review. JAMA 2014;311:1901-11.

4 Owsley C, Ball K, McGwin G, et al. Visual processing impairment and risk of motor vehicle crash among older adults. JAMA 1998;279:1083-8.

5 Johnson CA, Keltner JL. Incidence of visual field loss in 20,000 eyes and its relationship to driving performance. Arch Ophthalmol 1983;101:371-5.

6 McGwin G, Xie A, Mays A, et al. Visual field defects and the risk of motor vehicle collisions among patients with glaucoma. Invest Ophthalmol Vis Sci 2005;46:4437-41.

7 Haymes SA, Leblanc RP, Nicolela MT, et al. Risk of falls and motor vehicle collisions in glaucoma. Invest Ophthalmol Vis Sci 2007;48:1149-55.

8 Haymes SA, LeBlanc RP, Nicolela MT, et al. Glaucoma and on-road driving performance. Invest Ophthalmol Vis Sci 2008;49:3035-41.

9 Tanabe S, Yuki K, Ozeki N, et al. The association between primary open-angle glaucoma and motor vehicle collisions. Invest Ophthalmol Vis Sci 2011;52:4177-81.

10 McGwin G, Huisingh C, Jain SG, et al. Binocular visual field impairment in glaucoma and At-Fault motor vehicle collisions. J Glaucoma 2015;24:138-43.

11 Kwon M, Huisingh C, Rhodes LA, et al. Association between glaucoma and At-fault motor vehicle collision involvement among older drivers a population-based study. Ophthalmology 2016;123:109-16.

12 Yuki K, Asaoka R, Tsubota K. The relationship between central visual field damage and motor vehicle collisions in primary open-angle glaucoma patients. PLOS ONE 2014;9:e115572.

13 Kunimatsu-Sanuki $S$, Iwase A, Araie $M$, et al. An assessment of driving fitness in patients with visual impairment to understand the elevated risk of motor vehicle accidents. BMJ Open 2015;5:e006379.

14 Huisingh C, McGwin G Jr, Wood J, et al. The driving visual field and a history of motor vehicle collision involvement in older drivers: a population-based examination. Invest Ophthalmol Vis Sci 2014;56:132-8.

15 Glen FC, Smith ND, Crabb DP. Impact of superior and inferior visual field loss on hazard detection in a computer-based driving test. $\mathrm{Br} J$ Ophthalmol 2015;99:613-17

16 Coeckelbergh TR, Brouwer WH, Cornelissen FW, et al. The effect of visual field defects on driving performance: a driving simulator study. Arch Ophthalmol 2002;120:1509-16. 
17 Szlyk JP, Mahler CL, Seiple W, et al. Driving performance of glaucoma patients correlates with peripheral visual field loss. J Glaucoma 2005;14:145-50.

18 Bronstad PM, Bowers AR, Albu A, et al. Driving with central field loss I: effect of central scotomas on responses to hazards. JAMA Ophthalmol 2013;131:303-9.

19 Prado Vega R, van Leeuwen PM, Rendón Vélez E, et al. Obstacle avoidance, visual detection performance, and eye-scanning behavior of glaucoma patients in a driving simulator: a preliminary study. PLOS ONE 2013;8;e77294.

20 Mayhew DR, Simpson HM, Wood KM, et al. On-road and simulated driving: concurrent and discriminant validation. J Safety Res 2011;42:267-75.

21 Lee HC, Cameron D, Lee AH. Assessing the driving performance of older adult drivers: on-road versus simulated driving. Accid Anal Prev 2003;35:797-803.
22 Nelson-Quigg JM, Cello K, Johnson CA. Predicting binocular visual field sensitivity from monocular visual field results. Invest Ophthalmol Vis Sci 2000;41:2212-21.

23 Crabb DP, Fitzke FW, Hitchings RA, et al. A practical approach to measuring the visual field component of fitness to drive. Br J Ophthalmol 2004;88:1191-6.

24 Sumi I, Shirato S, Matsumoto S, et al. The relationship between visual disability and visual field in patients with glaucoma. Ophthalmology 2003;110:332-9.

25 Bhorade AM, Yom VH, Barco P, et al. On-road driving performance of patients with bilateral moderate and advanced glaucoma. Am J Ophthalmol 2016;166:43-51.

26 Weber J, Schultze T, Ulrich $\mathrm{H}$. The visual field in advanced glaucoma. Int Ophthalmol 1989;13:47-50. 